\title{
EDITORIAL
}

\section{What's in a name ... apropos MPO}

\author{
SC Lee
}

Center for Biomedical Engineering, 286 Bevis Hall, 1080 Carmack Road, Columbus, OH 43210, USA

Science is an communal endeavor of vast complexity, subtlety and importance to the human commonweal. As such, it behoves practitioners of the art to strive for clarity, and to communicate findings with alacrity, accuracy and without ambiguity. With regard to this last point, I draw attention to a paper published by my group in this issue of Leukemia (pp 1277-1285) describing phage display mutagenesis of a species of myelopoietin. The myelopoietins are a novel class of chimeric hematopoietic growth factors containing dual receptor agonists, which exploit synergistic effects of early and late acting factors in hematopoiesis (hIL-3 and hG-CSF receptor agonists, respectively $)^{1-3}$ to alleviate both neutropenia and thrombocytopenia following myelosuppression. ${ }^{4}$

As is customary, it fell to the inventors of the compounds to name these new candidate therapeutics, and the name they chose (myelopoietin) was unique, logical and descriptive, evoking the hematopoietic properties of the compounds, as well as the high potency they exhibited in regeneration of myeloid lineages. An abbreviation, MPO, inevitably followed christening the new class of compounds, perhaps influenced by similar abbreviations used for naturally occurring hematopoietic growth factors (TPO for thrombopoietin, EPO for erythropoietin). However, MPO is a less propitiously chosen abbreviation in that it is not unique in hematology, and therefore is ambiguous. MPO has for years been the widely accepted abbreviation for the neutrophil enzyme myeloperoxidase.

In light of the vastness of the scholarly endeavor, and the finite nature of language, it is almost inevitable that some nomenclatures occur more than once in various disciplines. In many cases, the context in which similar names occur is so disparate that confusion is minimal. For instance, confusion over whether the abbreviation 'APC' refers to 'antigen presenting cells' or to 'armored personnel carriers' is very likely to be quickly resolved with cursory examination of the publication in which the abbreviation occurs. However, it seems likely that persons with an interest in hematopoiesis might well be familiar with neutrophil function, and therefore think of MPO as referring to myeloperoxidase. The possibility of significant confusion and misinterpretation in the case of MPO referring to myelopoietin is real.

The abbreviation MPO has been the topic of multiple discussions between myself and the editors of Leukemia, and I accept their point that MPO is not an optimal abbreviation for our novel chimeric growth factors. I did not invent the myelopoietin class of compounds, and so it may not be my privilege to rename them (though suggested abbreviations such as 'MPoietin' are attractive). Still, in the spirit of clarity of scientific communication, neither MPO nor any other abbreviation for myelopoietin occurs in our manuscript. This avoids ambiguity in the work at hand, and leaves assignment of a new abbreviation to this class of compounds to more appropriate parties.

\section{References}

1 McWherter CA, Feng Y, Zurfluh LL, Klein BK, Baganoff MP, Polazzi JO, Hood WF, Paik K, Abegg AL, Grabbe ES, Shieh JJ, Donnelly AM, McKearn JP. Circular permutation of the granulocyte colony-stimulating factor receptor agonist domain of myelopoietin. Biochemistry 1999; 38: 4564-4571.

2 Mertlesmann R. Hematopoietic cytokines: from biology and pathophysiology to clinical application. Leukemia 1993; 7 (Suppl. 2): S168-S177.

3 Pebusque MJ, Fay C, Lafarge M, Sempere C, Saeland S, Caux C, Mannoni P. Recombinant human IL-3 and G-CSF act synergistically in stimulating the growth of leukemia cells. Leukemia 1989; 3: 200-205.

4 MacVittie TJ, Farese AM, Smith WG, Baum CM, Burton E, McKearn JP. Myelopoeitin, an engineered chimeric IL-3 and G-CSF receptor agonist, stimulates multilineage hematopoietic recovery in a nonhuman primate model of radiation-induced myelosuppression. Blood 2000; 95: 837-845. 